\title{
Evaluation of urinary antigen ELISA for diagnosing Legionella pneumophila serogroup 1 infection
}

\author{
R J Birtles, T G Harrison, D Samuel, A G Taylor
}

\begin{abstract}
The enzyme linked immunosorbent assay (ELISA) described was developed to detect a soluble antigen in the urine of patients with Legionnaires' disease caused by Legionella pneumophila serogroup 1 (L.pn 1). The assay was evaluated and showed good specificity $(100 \%)$ and intra-assay reproducibility. Antigen was detected in the urine of 93 (77\%) of 120 patients, overall, and in $86 \%$ of patients from whom a specimen obtained within seven days of onset of illness was available. On all but one occasion the first urine sample taken from a patient for whom a positive ELISA result was obtained, was itself positive. In one case antigen was not detected at four days but was present on the fifth day after onset of symptoms. In two patients urinary antigen was detectable as early as two days after onset of symptoms. In another the antigen persisted for at least 60 days. More than half the patients, however, had stopped producing detectable antigen within 14 days of onset of symptoms. It is therefore important that where Legionnaires' disease is suspected urine is collected as early as possible in the course of the disease.
\end{abstract}

At present the laboratory diagnosis of Legionnaires' disease in the United Kingdom is usually made either by culture of Legionella pneumophila from clinical material or by estimation of a patient's specific antibody response to the bacterium using the indirect fluorescence antibody test (IFAT) or the rapid microagglutination test (RMAT). Although a rapid diagnosis can often be established using either of these tests, the diagnosis can sometimes be delayed.

The diagnosis of Legionnaires' disease by the detection of legionella antigens in patients' urine using an ELISA was first described by Tilton, ${ }^{1}$ and several further assays have been reported. ${ }^{2-4}$ It is clear that the ability of such assays to detect antigens early in the course of the illness makes them potentially useful diagnostic tools. To date, however, no assay has been thoroughly evaluated and none is widely used in the United Kingdom. In this paper we describe an ELISA for the recognition of an L.pn 1 urinary antigen, using a fluoresceinisothiocyanate (FITC)-anti FITC system to amplify the signal. The ELISA was evaluated with respect to specificity, sensitivity, and reproducibility and the results were compared with those obtained using established diagnostic methods.

\section{Methods}

Hyperimmune rabbit antisera raised against the Philadelphia-1 strain of L.pn 1 (NCTC 11192) were produced as described by Harrison and Taylor. ${ }^{5}$ The immunoglobulin G portion of the immune serum was purified by affinity chromatography on a protein ASepharose column (Pharmacia, Sweden) using standard methods. The concentration of protein in the purified preparation was estimated by measuring optical density (OD) at $280 \mathrm{~nm}$.

A monoclonal antibody (LKC2b) was raised against the Knoxville-1 strain of L.pn 1 (NCTC 11286). The strain was grown on buffered charcoal yeast extract agar (BCYE) at $37^{\circ} \mathrm{C}$ for 48 hours. Cells were then removed into $0 \cdot 15 \mathrm{M}$ phosphate-buffered saline, $\mathrm{pH} 7 \cdot 2$ (PBS), containing $2 \%$ formalin, and incubated at $37^{\circ} \mathrm{C}$ overnight to kill the bacteria. The suspension was diluted to an OD at $605 \mathrm{~nm}$ equivalent to 1.2 and mixed at a ratio of $1: 1$ with Freund's incomplete adjuvant. $\mathrm{Balb} / \mathrm{c}$ mice were inoculated intraperitoneally on five occasions with $100 \mu \mathrm{l}$ of this preparation at fortnightly intervals. Spleens were removed three days after the final inoculation.

Spleen cells from the immunised mice were fused with mouse myeloma cell line, JK-Ag 8653, using standard methods. ${ }^{67}$ Culture supernatants from the hybridomas were screened against the Knoxville-1 strain by immunofluorescence. $^{5}$ Hybridoma cultures producing antibodies which showed good fluorescence were cloned by limiting dilution. Ascitic fluid was obtained by reintroducing one of the stable antibody secreting clones (LKC2b) into Balb/c mice treated with pristane.

The antibody isotype of LKC2b was determined by immunofluorescence using class and subclass specific conjugates (Tago, Burlingame, California, USA). The serogroup and subgroup specificity of the monoclonal antibody was determined by immunofluorescence, firstly with each of the 14 serogroups of $L$ pneumophila then with the 10 L.pn 1 subgroups. $^{8}$

LKC2b monoclonal antibody was purified by a modification of the euglobin precipitation method described previously. ${ }^{9}$ Briefly, the ascitic fluid was clarified by centrifugation, 
then 20 volumes of ice cold $2 \%$ boric acid were slowly added to one volume of ascitic fluid with stirring on ice for 30 minutes. The euglobin precipitate was recovered after centrifugation at $600 \times g$ for 20 minutes and resuspended in $2 \mathrm{ml}$ of $0 \cdot 1 \mathrm{M}$ carbonate buffer, $\mathrm{pH} 9 \cdot 2$, containing $0 \cdot 5 \mathrm{M} \mathrm{NaCl}$.

\section{ANTI-L.PN 1 FITC CONJUGATE}

FITC labelled antibody was prepared using methods similar to those described by Samuel et al for protein-FITC conjugates. ${ }^{10}$ Briefly, FITC ( $5 \mathrm{mg} / \mathrm{ml}$ in dry absolute ethanol) was added at a molar ratio of 20:1 to a stirred preparation of LKC2b IgM, diluted in $0 \cdot 1 \mathrm{M}$ carbonate buffer, containing $0.5 \mathrm{M} \mathrm{NaCl}(\mathrm{pH}$ $9 \cdot 2$ ) in the dark at room temperature. The reaction was allowed to continue for a further 60 minutes, then the LKC2b-FITC was separated from free FITC by gel filtration through Sephadex G25 using a PD 10 column (Pharmacia, Sweden) preblocked with $1 \%$ bovine serum albumin, and equilibrated with PBS. Fractions containing conjugate were pooled and stored in PBS containing 30\% (v/ v) glycerol and $0.08 \%$ azide.

The production and characterisation of the anti-FITC monoclonal antibody (2F3.1) used in this study has been described previously. ${ }^{10}$ A horseradish peroxidase (EC1.11.1.7) conjugate of this antibody was prepared by the periodate method. ${ }^{11}$

\section{URINARY ANTIGEN ELISA}

Flat bottomed microtitre plates (NuncImmuno Plate 1) were coated with capture antibody by adding $200 \mu \mathrm{l}$ of purified rabbit IgG $(10 \mu \mathrm{g} / \mathrm{ml})$ in PBS ( $\mathrm{pH} 7 \cdot 2)$ containing $0.08 \%(\mathrm{w} / \mathrm{v})$ sodium azide to each well. Plates were sealed and incubated at $20^{\circ} \mathrm{C}$ for 48 hours before being stored at $4^{\circ} \mathrm{C}$ until use.

Before use the wells were washed four times with PBS containing $0.1 \%(\mathrm{v} / \mathrm{v})$ Tween 20 (PBS-Tween). Urine to be tested was heated in a boiling waterbath for 10 minutes, cooled, then centrifuged in a bench centrifuge at $10000 \times g$ to remove any precipitate. Urine $(100 \mu \mathrm{l})$ was added to each well and the plate incubated at $37^{\circ} \mathrm{C}$ for 60 minutes, then washed four times in PBS-Tween. LKC2bFITC $(90 \mu \mathrm{l})$, diluted $1 / 800$ in PBS-Tween containing $5 \%$ horse serum (HS), was then added to each well and the plate was incubated as above. After further washing 85 $\mu l$ of the anti-FITC horseradish peroxidase conjugate, diluted 1/3000 in PBS-Tween-HS, was added to each well followed by an incubation and washing step as above.

Substrate was prepared by diluting a 10 $\mathrm{mg} / \mathrm{ml}$ stock solution of 3,3', 5,5' tetramethylbenzidine in dimethyl-sulphoxide to $100 \mu \mathrm{g} /$ $\mathrm{ml}$ in $0.1 \mathrm{M}$ citrate-acetate buffer ( $\mathrm{pH} 6.0$ ) containing $0.006 \%(\mathrm{v} / \mathrm{v})$ hydrogen peroxide. To each well was added $100 \mu l$ to detect bound anti-FITC horseradish peroxidase and after a final 30 minute incubation the reaction was stopped with $50 \mu \mathrm{l}$ of $2 \mathrm{M}$ sulphuric acid. The intensity of the subsequent yellow colour produced was measured spectrophotometrically at $450 \mathrm{~nm}$ wavelength $\left(\mathrm{OD}_{450}\right)$ using a plate-reader blanked on a coated well containing only substrate.

Three control preparations were used in each assay. These consisted of two positive control urine specimens, A and B, collected from patients with confirmed Legionnaires' disease, and a negative control urine sample, C, collected from an apparently healthy member of staff. For a dose-response study urine samples from controls A and B were diluted with distilled water to concentrations ranging between $10 \%$ and $85 \%(v / v)$ of the neat urine, then each concentration was tested in quadruplicate in the ELISA.

\section{PATIENT SPECIMENS}

Urine specimens from 120 confirmed cases of Legionnaires' disease were available for evaluation of the diagnostic sensitivity of the ELISA. For inclusion in the evaluation Legionnaires' disease was confirmed using one of the diagnostic criteria outlined in table 1.

Where possible, the following information was also obtained: (1) date of onset of symptoms; (2) dates of collection of the urine samples; (3) dates of collection of serum samples and the IFAT results obtained; (4) the subgroup of L.pn 1 isolated; (5) date of exposure to infectious source (outbreak situations only).

Urine samples from 80 patients diagnosed as having an infection due to an agent other than Legionella were available for evaluation of the specificity of the ELISA (table 2). The specimens were obtained from two sources.

(i) Forty seven urine samples (one from each patient) were kindly given by the British Thoracic Society (BTS). These urines had been collected from patients with pneumonia as part of a prospective study organised by the BTS and the Public Health Laboratory Service

Table 1 Evidence of infection in 120 patients with confirmed Legionnaires' disease and sensitivity of ELISA in these cases

\begin{tabular}{lcccc}
\hline Diagnosis established by: & Number of cases & $\begin{array}{l}\text { Antigen } \\
\text { positive }\end{array}$ & $\begin{array}{l}\text { Antigen } \\
\text { negative }\end{array}$ & Sensitivity \\
\hline Isolation of Legionella pneumophila serogroup 1 & 51 & 43 & 8 & $84 \%$ \\
Diagnostic serology & 47 & 33 & 14 & $70 \%$ \\
Raised antibody titres and outbreak association & 22 & 17 & $77 \%$ & $77 \%$ \\
Total & 120 & 93 & 27 & 7 \\
\hline
\end{tabular}

^At least one urine sample taken from each patient was positive when tested in the L.pn 1 urinary ELISA

†None of the patient's urine samples was positive when tested in the ELISA.

${ }_{+}^{+}$Fourfold rise in IFAT titres to at least 64 or standing titre of 128 . 
Table 2 Details of infection and diagnosis for 80 patients with non-L.pn 1 pneumonia of known aetiology

\begin{tabular}{|c|c|c|c|}
\hline Aetiological agent & $\begin{array}{l}\text { Source of } \\
\text { specimen }\end{array}$ & Method of diagnosis & $\begin{array}{l}\text { Number of } \\
\text { patients }\end{array}$ \\
\hline \multirow[t]{4}{*}{ Streptococcus pneumoniae } & BTS $^{1}$ & $\begin{array}{l}\text { Isolation from blood } \\
\text { Isolation from sputum }\end{array}$ & $\begin{array}{l}10 \\
14\end{array}$ \\
\hline & EBTS $^{2}$ & Blood culture & 1 \\
\hline & & Sputum culture & 9 \\
\hline & $\mathbf{L R U}^{3}$ & Evidence not stated 4 & 1 \\
\hline \multirow[t]{2}{*}{ Mycoplasma pneumoniae } & BTS & Serology 5 & 11 \\
\hline & LRU & Serology & 3 \\
\hline \multirow{7}{*}{$\begin{array}{l}\text { Klebsiella pneumoniae } \\
\text { Haemophilus influenzae } \\
\text { Salmonella virchow } \\
\text { Streptococcus spp } \\
\text { Aerobic Gram negative bacilli } \\
\text { Influenza A virus }\end{array}$} & LRU & Evidence not stated & 1 \\
\hline & LRU & Evidence not stated & 1 \\
\hline & LRU & Isolation & 1 \\
\hline & BTS & Evidence not stated & 1 \\
\hline & BTS & Evidence not known & 2 \\
\hline & $\begin{array}{l}\text { BTS } \\
\text { LRU }\end{array}$ & $\begin{array}{l}\text { Serology } \\
\text { Serology }\end{array}$ & $\begin{array}{l}3 \\
3\end{array}$ \\
\hline & & Evidence not stated & 2 \\
\hline \multirow{7}{*}{$\begin{array}{l}\text { Influenza B virus } \\
\text { Varicella zoster virus } \\
\text { Cytomegalovirus } \\
\text { Coxiella burnetii } \\
\text { Pneumocystis carinii } \\
\text { Chlamydia psittacii }\end{array}$} & LRU & Serology & 1 \\
\hline & BTS & Evidence not stated & 1 \\
\hline & LRU & Evidence not stated & 1 \\
\hline & BTS & Serology & 2 \\
\hline & LRU & Direct immunofluorescence on sputum & 8 \\
\hline & BTS & Serology & 3 \\
\hline & LRU & Evidence not stated & 1 \\
\hline
\end{tabular}

1 British Thoracic Society and Public Health Laboratory Service study.

2 Patients with pneumonia excluded from the final BTS evaluation.

3 Specimens sent to the PHLS Legionella Reference Unit.

4 Diagnosis confirmed by submitting laboratory but evidence not stated.

5 Fourfold rise in titres or high conversion titres.

6 Patient had fever of unknown origin and septicaemia.

(PHLS) in 1982-83. ${ }^{12}$ The aetiological agent was known for each patient's infection. Urine samples from a further 10 patients with laboratory evidence of pneumococcal pneumonia were also obtained from the BTS, but these patients had been excluded from their study.

(ii) Twenty three urine samples (one from each patient) were submitted to the PHLS Legionella Reference Unit (LRU) from patients with possible legionella infections, but a diagnosis other than Legionnaires' disease was subsequently established.

Urine was also available from three patients with Legionnaires' disease confirmed by culture caused by non-Sgp $1 L$ pneumophila. These cases were shown to have infection caused by $L$ pneumophila serogroups 3,8 , and 12 , and their urine samples were examined to determine the serogroup specificity of the ELISA.

\section{REPRODUCIBILITY}

Statistical evaluation of within batch and between batch reproducibility was carried out as described by Strike. ${ }^{14}$ Briefly, 10 urine samples (including negative, weakly positive, strongly positive and control urines $A, B$, and $C$ ) were randomly allocated to microtitre wells and tested in duplicate in the same microtitre plate. The mean and standard deviation of each pair of $\mathrm{OD}_{450}$ readings was determined for each urine sample. The ELISA was then repeated a further nine times over a period of days. These data were then used for the calculation of the within batch standard deviation $\left(S_{\mathrm{w}}\right)$ and the between batch standard deviation $\left(S_{b}\right)$.

Initially an $S_{w}$ was calculated as described above but only using data for control urines A, $B$, and C. The $S_{w}$ was then updated after each batch of new results using the standard deviations of control urines $\mathrm{A}, \mathrm{B}$, and $\mathrm{C}$ from the 10 most recent assays. Acceptance or rejection of a batch of results was decided using the criteria outlined by Strike. ${ }^{13}$

\section{Results}

The isotype of LKC2b monoclonal antibody was determined to be IgM. When tested by immunofluorescence against the 10 subgroups of L.pn 1, LKC2b reacted with representative strains from the "Philadelphia", "Allentown", "Benidorm", "Knoxville" and "France" subgroups of L.pn 1; these are the same subgroups recognised by $2 \mathrm{Mab}^{11}$ of the International panel. ${ }^{8}$

A dose-response curve determined using dilutions of control urine $A$ is shown in the figure. The coefficients of variation (CV) calculated from quadruplicate samples of each dilution are shown in table $3 .{ }^{13}$ Also shown in the figure is a dose-response curve for control B; both curves seem to be parallel, although statistical confirmation was not attempted.

\section{REPRODUCIBILITY}

The $S_{w}$ was found to be very low $(0.051$ OD units within a possible range of values between 0.0 and 2.0 ), allowing small differences in the concentration of urinary antigen to be determined with confidence. The $S_{b}$, however (calculated for each of the three control urine samples) were found to be too high to allow antigen concentrations to be compared from one plate to another (table 4). Results were

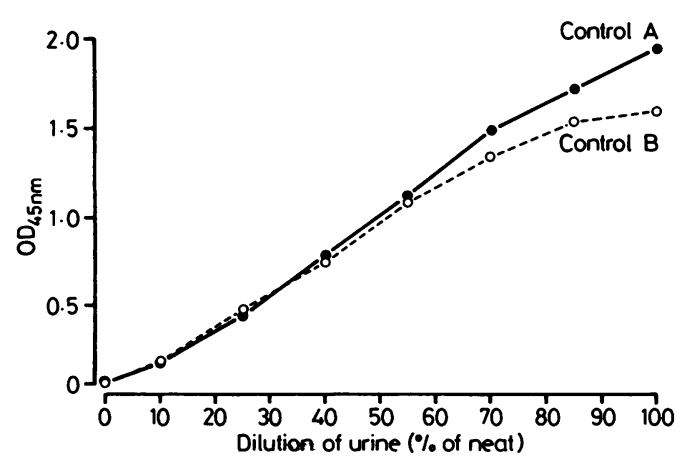

Dose-response curves for control urines $A$ and $B$. 
Table 3 Coefficient of variation ( $C V \%$ ) for dilutions of control A urine

\begin{tabular}{|c|c|c|c|}
\hline $\begin{array}{l}\text { Concentration of urine } \\
\left({ }_{0}^{\circ}, v / v \text { of neat }\right)\end{array}$ & Mean $O D_{450}$ & Standard deviation & $\mathrm{CV} \%$ \\
\hline $\begin{array}{r}100 \\
85 \\
70 \\
55 \\
40 \\
25 \\
10 \\
0\end{array}$ & $\begin{array}{l}1.94 \\
1.71 \\
1.48 \\
1.12 \\
0.78 \\
0.43 \\
0.17 \\
0.05\end{array}$ & $\begin{array}{l}0.08 \\
0.09 \\
0.08 \\
0.10 \\
0.09 \\
0.05 \\
0.02 \\
0.01\end{array}$ & $\begin{array}{r}4.1 \\
5.3 \\
5.4 \\
8.9 \\
11.5 \\
11.6 \\
11.8 \\
14.0\end{array}$ \\
\hline
\end{tabular}

therefore only compared when determined within the same batch.

\section{DISCRIMINATION BETWEEN POSITIVE AND}

NEGATIVE RESULTS

If the $\mathrm{OD}_{450}$ (mean of duplicate estimation) of the urine being examined is greater than the $\mathrm{OD}_{450}$ of the negative control $\mathrm{C}+2 \cdot 77 \mathrm{~S}_{\mathrm{w}}$ then the urine was regarded as positive. In practice most results could easily be determined by eye.

\section{SPECIFICITY}

The aetiological agents responsible for disease in 80 patients known to have infection other than L.pn 1 pneumonia are shown in table 2. All the samples from these patients gave negative results in the urinary antigen ELISA and the specificity was therefore calculated to be $100 \%$. In preliminary experiments a false positive reaction was obtained on one occasion when a urine specimen had not been boiled and clarified by centrifugation before being tested.

Three patients had Legionnaires' disease caused by $L$ pneumophila of serogroups other than serogroup 1. These patients produced no detectable antigen in their urine, suggesting the assay detects an antigen which is serogroup specific.

\section{DIAGNOSTIC SENSITIVITY}

At least one urine specimen was available from each of 120 patients with confirmed Legionnaires' disease. L.pn 1 urinary antigen was detected in 93 of $120(77 \%)$ of these patients (table 1).

The date of onset of symptoms was known for 112 of 120 patients. Altogether, 202 urine samples were collected from these patients at various dates during their illness. The diagnostic sensitivity of the assay decreased as the time interval between onset of symptoms and the date of urine collection increased (table 5). The sensitivity was significantly higher $(86 \%)$ when testing urines which had been collected within seven days of onset of symptoms than testing urines collected after this time $(64 \%)$ $\left(\chi^{2}=3.90,0.01<p<0.05\right)$. It is noteworthy that when antigen was detected in the

Table 4 Between assay standard deviation $\left(S_{b}\right)$ of control urine samples

\begin{tabular}{lcl}
\hline Control urine sample & Mean $O D_{450}$ & $S_{b}$ value \\
\hline Positive A & 1.133 & 0.224 \\
Positive B & 0.478 & 0.212 \\
Negative C & 0.095 & 0.058 \\
Possible range of $\mathrm{OD}_{450}$ values $=0.0-2.0$ & \\
\hline
\end{tabular}

ELISA, with one exception, it was always present in the earliest available specimen.

Urine specimens giving a positive ELISA result were obtained from patients with Legionnaires' disease confirmed by isolation of the following L.pn 1 subgroups: "Philadelphia"; "Benidorm"; "Knoxville"; "France"; and "Allentown". Urine from one patient with Legionnaires' disease confirmed by isolation of L.pn 1 subgroup "Camperdown" contained no detectable urinary antigen, but this sample was collected 22 days after onset of symptoms when the sensitivity of the ELISA is reduced.

ONSET AND DURATION OF ANTIGEN EXCRETION

Eighty seven patients with Legionnaires' disease with known onset dates had at least one urine sample collected which gave a positive result when tested in the ELISA. Urine samples which contained detectable quantities of urinary antigen were collected as early as two days after onset of patient symptoms.

Fifteen of these 87 patients produced urine samples at later dates which did not contain detectable antigen and these patients provided information about the duration of antigen excretion (table 6). The first urine samples which were negative when tested in the ELISA were collected from these patients between seven and 90 days after onset of symptoms. Eight of the 15 patients were producing urine which contained no detectable antigen within 14 days of the onset of their symptoms. Antigen was still detected in urine of one of the 87 patients 60 days after onset of symptoms.

The onset of antigen excretion was also related to the time after exposure to the source of infection. Detailed epidemiological investigations showed a period of three days as the probable period of exposure to $L$ pneumophila in a recent outbreak. ${ }^{14}$ The dates of collection of 79 urine samples from 41 patients infected in this outbreak were related to the earliest of these dates. The shortest interval between exposure and collection of urine was 10 days; urine taken at this time was available from two patients and both specimens contained detectable urinary antigen.

SEROLOGICAL RESPONSE OF PATIENTS EXCRETING URINARY ANTIGEN

The dates of collection and IFAT results were known for 53 serum samples from 22 patients who were urinary antigen positive within seven days of onset of symptoms (table 7). Only two of 22 of the samples showed diagnostic titres in IFAT within seven days of onset of symptoms. All sera taken between eight and 14 days after 
Table 5 Sensitivity of ELISA in relation to time after onset of symptoms ${ }^{\star}$

\begin{tabular}{llllll}
\hline & \multicolumn{7}{l}{ Number of days after onset of symptoms } \\
\cline { 2 - 6 } & $01-07$ & $08-14$ & $15-21$ & $22-28$ & $>28$ \\
\hline Total number of urine samples & 42 & 82 & 33 & 20 & 25 \\
Number of positive urine samples & 36 & 57 & 23 & 14 & 8 \\
Sensitivity & $86 \%$ & $69 \%$ & $70 \%$ & $70 \%$ & $32 \%$ \\
\hline
\end{tabular}

onset had positive titres, and 14 of 19 of these had diagnostic titres. Of the remaining sera taken 15 days or more after onset of symptoms, 10 of 12 gave diagnostic titres. Nineteen of the 22 patients were diagnosed serologically. The remaining three patients were diagnosed by isolation of L.pn 1 from their respiratory tract, but sera collected from all three early in their illnesses showed positive rising titres. No convalescent sera were availale from these patients.

\section{Discussion}

The ELISA described here uses a monoclonal antibody to detect the captured antigen. This approach has advantages over most previously reported $L$ pneumophila urinary antigen ELISAs which use polyclonal antisera. ${ }^{134}$ Firstly, the monoclonal antibody can be prepared in large quantities which overcomes problems of reproducibility encountered when preparing polyclonal antisera. ${ }^{15}$ Secondly, FITC conjugates of LKC2b are easily prepared and do not give rise to problems of non-specific binding. The limitation of using LKC2b is that it only reacts with the L.pn 1 subgroups recognised by the "Mab 2" antibody of the international panel. ${ }^{8}$ Although this specificity was only shown by immunofluorescence, it might be expected that it would also apply to the ELISA. This is supported by the observation that a urine sample from a patient infected with a strain of the L.pn 1 subgroup "Camperdown" (not reactive with LKC2b by immunofluorescence) was negative when tested in the ELISA. Previous studies indicate that over $95 \%$ of all cases of Legionnaires' disease are caused by L.pn $1,{ }^{16}$ of which about $90 \%$ are caused by strains belonging to subgroups reactive with LKC2b. ${ }^{17}$ All outbreaks of community acquired Legionnaires' disease in the United Kingdom have also been

Table 6 Duration of detectable antigen in 15 confirmed cases of Legionnaires' disease

\begin{tabular}{lll}
\hline & $\begin{array}{l}\text { Last day } \\
\text { positive urine sample } \\
\text { was taken }\end{array}$ & $\begin{array}{l}\text { First day that a } \\
\text { negative urine sample } \\
\text { was taken }\end{array}$ \\
\hline 1 & 3 & 7 \\
2 & 5 & 7 \\
3 & 5 & 13 \\
4 & 6 & 11 \\
5 & 8 & 9 \\
6 & 8 & 10 \\
7 & 9 & 11 \\
8 & 11 & 12 \\
9 & 13 & 90 \\
10 & 14 & 17 \\
11 & 15 & 30 \\
12 & 22 & 28 \\
13 & 27 & 45 \\
14 & 30 & 32 \\
15 & 31 & \\
\hline
\end{tabular}

^Number of days after onset of symptoms.
Table 7 Serological results obtained for 53 serum specimens from 22 patients who produced detectable urinary antigen within seven days of onset

\begin{tabular}{lccc}
\hline & \multicolumn{3}{c}{ Days after onset of symptoms } \\
\cline { 2 - 4 } Serolog $y^{\star}$ & $00-07$ & $08-14$ & $>14$ \\
\hline Negative & 16 & 0 & 1 \\
Positive but not diagnostic & 4 & 5 & 1 \\
Diagnostic & 2 & 14 & 10 \\
Total (Sera/patients) & $22 / 17$ & $19 / 16$ & $12 / 8$ \\
\hline
\end{tabular}

${ }^{\star}$ Results as defined by Harrison and Taylor. ${ }^{21}$.

caused by L.pn 1 subgroups recognised by LKC2b (unpublished observations); thus the insensitivity of the LKC2b to the nonserogroup $1 L$ pneumophila serogroups and possibly some L.pn 1 subgroups is not a major disadvantage.

The use of the FITC-anti-FITC amplification system in the ELISA has several potential benefits. This system has been shown to be a very sensitive method of detecting proteins in both ELISA and dot blots and may be preferable to other enhancement systems because the conjugates are easy to prepare and the antiFITC enzyme conjugate exhibits very low non-specific binding. ${ }^{10} 18$

A dose-response curve was shown for the ELISA using positive controls $A$ and $B$, indicating that quantification of the concentration of antigen in a test urine is possible. In the form presented here the ELISA is a qualitative test used to determine the presence or absence of antigen, but proposed work on the nature of the antigen being detected and the timing and relevance of its concentration may permit future quantification of the ELISA.

The reproducibility of the ELISA was very good where specimens were examined within the same batch. The comparison of $\mathrm{OD}_{450}$ values using statistical methods was essential during the evaluation of this assay, but in practice for most urines tested in the ELISA the difference between a positive and negative result was distinct enough to be determined by eye. The between batch reproducibility was poor, prohibiting comparison of results obtained in different assay batches. Problems with between batch reproducibility are thought to arise from the deterioration and variation of consumable reagents used in the ELISA, specifically horse serum and hydrogen peroxide. This limitation is not relevant, however, as the assay in its present form is designed for acute phase diagnosis of Legionnaires' disease from single samples only, and there is no necessity to compare different urine samples over a series of ELISA batches.

The specificity of the ELISA was calculated to be $100 \%$ as no false positive results were obtained using urine samples from patients with non-L.pn 1 pneumonia. This estimate must be treated with caution as urine samples from only 80 patients were available for examination and the time at which these urines were collected, in relation to the patients' illnesses, was unknown. It is clear, however, that the ELISA is highly specific. As Legionnaires' disease accounts for only about $2 \%$ of all 
cases of pneumonia in the United Kingdom, ${ }^{12}$ this high specificity is very impontant as even a low percentage of false positive results would greatly reduce the predictive value of a positive result. $^{19}$

The overall diagnostic sensitivity of the ELISA was estimated to be about $77 \%$, a figure similar to those reported for previously described ELISAs ${ }^{15}$ and serological tests. ${ }^{20}$ The sensitivity of the ELISA, however, was shown to be optimal ( $86 \%$ ) during the first seven days of illness. It is therefore clear that a urine sample should be examined from a suspected case as soon as possible after onset of symptoms. The sensitivity of the ELISA decreased to about $70 \%$ when the urine samples tested were taken between the eighth and twenty eighth days of illness, and there was another significant decline in sensitivity when testing urines taken at any later times. The role of the ELISA in late or retrospective diagnosis of Legionnaires' disease is therefore limited. This is in contrast to serology where high titres of antibody often persist for a prolonged period. ${ }^{21}$

In two patients urinary antigen became undetectable within seven days of onset of symptoms, and eight of 15 patients had stopped producing detectable levels of antigen within 14 days of onset. The duration of antigen excretion, however, is clearly very variable and in the case of one patient remained positive for at least 60 days after onset of symptoms. These observations are similar to those of Kohler et $a l^{4}$ who also showed that the duration of urinary antigen excretion was extremely variable in patients with Legionnaires' disease, ranging from four to 327 days.

In only one instance did a patient whose first urine sample was negative when tested in the ELISA excrete detectable levels of antigen in a subsequent urine sample. Even in this case antigen was detectable in urine taken on the fifth day after onset of the patient's symptoms. Thus if patients are not producing urine in which antigen can be detected within the first week of illness, they are probably unlikely to do so at all and "follow up" samples need not be collected. The validity of this conclusion will need further investigation.

The potential use of an ELISA to detect antigen in the urine of patients during the acute phase of Legionnaires' disease has been discussed for some time. ${ }^{1-3}$ The results of this study realise this potential with a rapid, non-invasive assay which has been evaluated using specimens from a large number of patients. The ELISA described is relatively simple to perform and results can be available within four hours of the sample being collected. Large numbers of urines can be screened simultaneously making the test particularly suitable for use in an outbreak situation. Microtitre plates can be coated and stored for at least nine months before use without evidence of deterioration. The ability of the ELISA to detect antigen during the very acute stages of infection (as soon as two days after onset of symptoms in some patients) make it a valuable addition to existing diagnostic tests. During a recent outbreak of community acquired Legionnaires' disease the urinary antigen ELISA was the first laboratory assay to identify L.pn 1 infection in 12 of 43 cases (unpublished observations).

We thank Mrs N Doshi for her help in the production of the monoclonal and polyclonal antibodies, the British Thoracic Society for providing urine specimens, and the staff of the Communicable Disease Surveillance Centre for epidemiological data.

1 Tilton RC. Legionnaires' disease antigen detected by enzyme-linked immunosorbent assay. Ann Intern Med 1979;90:697-8.

2 Bibb WF, Arnow PM, Thacker L, McKinney RM. Detection of soluble Legionella pneumophila antigens in serum and urine specimens by enzyme-linked immunosorbent assay with monoclonal and polyclonal antibodies. J Clin Microbiol 1984;20:478-82.

3 Sathapatayavongs B, Kohler RB, Wheat LJ, et al. Rapid diagnosis of Legionnaires' disease by urinary antigen detection. Am J Med 1982;72:576-82.

4 Kohler RB, Winn WC, Wheat LJ. Onset and duration of urinary antigen excretion in Legionnaires' disease. $J$ Clin Microbiol 1984;20:605-7.

5 Harrison TG, Taylor AG. Identification of Legionellae by serological methods. In: Harrison TG, Taylor AG, eds. $A$ laboratory manual for Legionella. Chicester: J Wiley and Sons Ltd, 1988:57-68.

6 Kohler G, Milstein C. Continuous cultures of fused cells secreting antibody to predefined specificity. Nature 1975;256:495-7.

7 Tedder RS, Yao JL, Anderson MS. The production of monoclonal antibodies to Rubella haemagglutinin and their use in antibody capture assays for Rubella specific IgM. J Hyg 1982;88:335-50.

8 Joly JR, Mckinney RM, Tobin JC, Bibb WF, Watkins ID, Ramsay D. Development of a standardised subgrouping scheme for Legionella pneumophila serogroup 1 using monoclonal antibodies. J Clin Microbiol 1986;23:768-71.

9 Masseyeff R, Gombert J, Josselin J. Methode de preparation de la $B_{2}$-macroglobuline du serum humain. Immunochemistry $1965 ; 2: 177-80$.

10 Samuel D, Patt RJ, Abuknesha RA. A sensitive method of detecting proteins on dot and Western blots using monoclonal antibody to FITC J Immunol Methods 1988, 107:217-24.

11 Wilson MB, Nakane PK. Recent developments in the periodate method of conjugating horseradish peroxidase HRPO) to antibodies. In: Knapp W, Holubar K, Wick G, eds. Immunofluorescence and related staining techniques. Amsterdam: Elsevier, 1978:215-24.

12 British Thoracic Society Research Committee. Communityacquired pneumonia in adults in British hospitals in 19821983: a survey of aetiology, mortality, prognostic factors 1983: a survey of aetiology, mortality, pro

13 Strike PW. Medical laboratory statistics. Bristol: John Wright and Sons Ltd, 1981:73-95.

14 Westminster Action Committee. Broadcasting House Legionnaires' Disease. London: Department of Environmental Services, City of Westminster, 1988:58-69.

15 Kohler RB, Sathapatayavongs B. Recent advances in the diagnosis of serogroup 1 Legionella pneumophila pneumonia by detection of urinary antigen. Zentralb Bakteriol Mikrobiol Hyg 1983;255 (1 Orig A):102-7.

16 Dournon E, Mayaud C, Bure A, Desplaces N, Christol D. Epidemiological features of Legionnaires' disease in the Paris area. Zentralbl Bakteriol Mikrobiol Hyg 1983;255 (1 Orig A):76;83.

17 Dournon E, Bibb WF, Rajagopalan P, Desplaces N, McKinney RM. Monoclonal antibody reactivity as a virulence ney RM. Monoclonal antibody reactivity as a virulence marker for Legionella pneum

18 Harmer IJ, Samuel D. The FITC-anti-FITC system is a sensitive alternative to biotin-streptavidin in ELISA. Immunol Methods 1989;122:115-21.

19 Vecchio TJ. Predictive value of a single diagnostic test in unselected populations. N Engl J Med 1966;274:1171-3.

20 Harrison TG, Dournon E, Taylor AG. Evaluation of sensitivity of two serological tests for diagnosing pneumonia caused by Legionella pneumophila serogroup 1. J Clin Pathol 1987;40:77-82.

21 Harrison TG, Taylor AG. Diagnosis of Legionnaires disease by antibody levels. In: Harrison TG, Taylor AG eds. A Laboratory manual for Legionella. Chichester: Wiley and Sons Ltd, 1988:115-9. 